\title{
Parametric design of a complex part in a FEM environment
}

\author{
Mădălina Ioana Blaj ${ }^{1, *}$, and Gheorghe Oancea ${ }^{1}$ \\ ${ }^{1}$ Transilvania University of Brasov, Department of Manufacturing Engineering, Mihai Viteazu, No. 5, \\ Brasov, Romania
}

\begin{abstract}
In the recent years, the industry tends to an accelerated evolution in order to maintain the competitiveness on the profile market. As a response to this challenge, engineers and researchers have started to combine multidisciplinary knowledge with solutions provided by programming software so as to obtain immediate and reliable results on each step of a product development. The main advantage is highlighted especially for the optimization phases, when the using of the parametric tools is less time-consuming and the majority of issues of the developed product are solved from the first version of the project. This paper aims to present a case study of a parametric design and meshing in MSC Patran environment, using a programming solution in Visual Basic, of a part with a complex geometry from aerospace industry and also to accentuate the minimized risks from the design phase to manufacturing, with a review of the advantages and disadvantages.
\end{abstract}

\section{Introduction}

Nowadays, the industrial companies are focusing on acquiring a place in the customer's preferences, their product launching on the profile market being distinguished by others with a short time of development and with various specifications. In order to exceed the customers' expectations regarding low-cost and high quality products, companies are searching, testing and implementing with achievements various solutions in each step of a product development.

Since the Tippett's contributions for work sampling in the textile industry, mathematical models for cost reductions from the 1980s [1] to the latest methods as lean principles and techniques, the companies are improving their work methods in order to withstand on the market. Lean principles and techniques constitute a philosophy, having the main purpose and benefits as lower production costs with qualitative output, into a reduced period of time.

The lean principles are the following: standardization, simplified and specified pathways, teaching and learning, socialization, continuous improvement, supplier-customer relationship, coordination through communication, expertise and stability, common goal establishment and knowledge sharing [2].

* Corresponding author: madalina.blaj@unitbv.ro 
Based on these principles, each phase of a product development can be improved, leading to the expected results. Considering the first step of a product development, the design phase is the essential step where the product is defined from the point of view of aspect, requirements, performances, using, manufacturing and maintainability. One method of work which implies many lean principles is to combine multidisciplinary knowledge since the starting point of a project, in order to obtain a parametric working approach [3].

If the appliance range is reduced to the civil, industrial, aerospace, automotive and adjacent domains, the parametric design is defined by the algorithms that define the relations between the elements which are compiling complex structures. The changing of the variables will generate new characteristics of the structure, offering many possibilities for optimisation procedures $[4,5]$.

Considering that the modelling of a product and its finite elements analysis are correlated and a base model of the product is completely developed, the paper purpose is to highlight the usefulness of the parameterization of the two steps from a product development.

Modelling of a product is referring to the $3 \mathrm{D}$ representation of the part - in this step is represented the geometric model of the part. Finite element analysis is an evaluation method of solutions of the mathematical equations which describes different phenomena with its limitations, in different domains [6 - 9].

\section{Parametric modelling and meshing of industrial products - advantages and disadvantages}

Compared with the classical working methods, the modelling and meshing parameterizations offer noticeable advantages, which are summarized below [4]:

- Detailed knowledge of the project - from the base project all the strong and weak points are known, also the needs and the difficulties, the time for documentation being reduced;

- Objectives set of the parametric design;

- Full control over the geometry - an infinite number of solutions are generated, offering the possibility to identify new optimisation areas;

- Creative ideas are encouraged - from the geometric layout to the scripts;

- Well-known and preferential established parameters - based on requirements, the optimum solution is chosen.

- Testing and evaluating of proposals based on different criteria established by requirements or customers;

- Easier issues and limitation identification;

- Reduced errors from the pre-processing step of the finite element analysis;

- Reduced time for creating a new CAD and meshing model;

- The manufacturing process is rushed;

- More finite element analysis are realised in a shorten period of time - as a result, more iterations from the optimisation phases are performed;

- Reduced costs for the overall project.

- Reduced human errors.

Also, this method implies disadvantages [4]:

- Suitable for mass production - implies time for advantageousness studies ;

- Implies engineering and programming knowledge;

- The user's creativity is reduced by the limitations imposed by creator;

- Long time for implementation and certification. 


\section{Software tool used for parametric designing}

In general, in order to create a parametric design of a part the designer has to transpose clearly the requirements of the software tool, which must be understandable for the users.

Initially, for the modelling step, the designer who will write the programming code has to study the part which will be parameterized (dimensions, geometry) and also its limitations regarding the geometry, location and manufacturing possibility. Considering the required output (e.g. interested modified dimensions), will be created an overview of the main parameters and also to all the steps which will be adapted for the digital part, in order obtain the real part, with different shapes and dimensions. In the end of this step the designer has to transpose the geometry creation in mathematical equations and then in programming code with all the necessary parameters which will describe the new part, based on required parameters.

As a second step, the designer will determine the meshing limitations of the part and to create a list with messages for the tool which will display to the user to try other values for the parameters. For meshing, auxiliary steps can be performed, as premeshing and node equivalence in order to avoid meshing errors (e.g. distorted elements, double nodes, curvature approximation). In the end for the step, the designer can establish the output e.g. 2D meshing or 3D meshing, with different typed of elements. For this step, the parameters could be the elements type and also the element size.

Finally, further checking has to be performed in order to identify possible issues with the tool - e.g. not all the limitations are covered, traceability is missing, not enough understanding for the user. After this step, the tool can be released and used for mass applications.

In time, after a period of using there is possibility to receive feedback from the software tool users in order to perform further updates of the software tool regarding the ease of using and also to correct identified issued regarding mathematical transposing of the parameters or of the output methods.

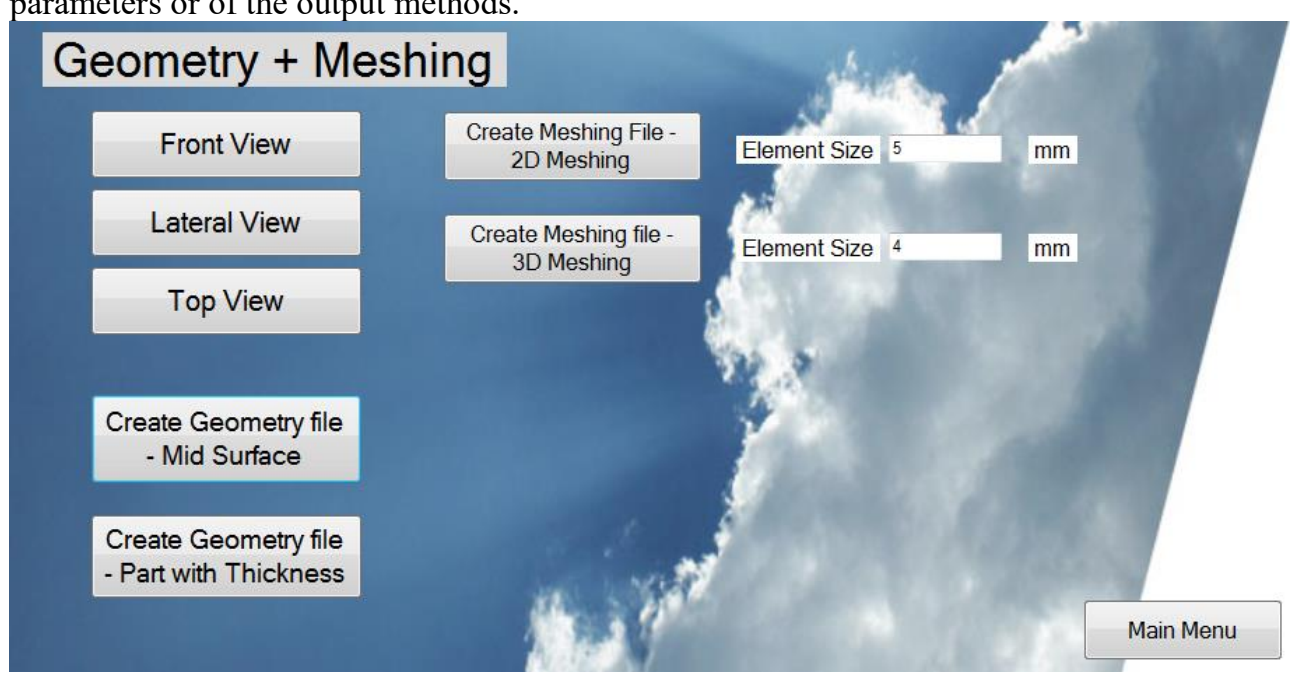

Fig. 1. Software tool interface - main window

\section{Parametric design of a complex part in a FEM environment. A case study}

The case study developed by the authors implies the modelling and meshing parameterization of a complex part from the aerospace industry, the result being a software 
tool created in Visual Basic with a friendly user interface (Fig. 1). The used FEM environment is MSC Patran software, which has the programming source code in PCL Patran Command Language.

The considered part is an example of a latch from the cargo door area for an aircraft. The cargo doors are designed to resist to the circumferential loads resulted especially after the aircraft pressurisation. The loads are transmitted to upper side of the door area by the door itself and the load path is continuing to the bottom side of door surrounding. In this location the considered part is located (Fig. 2). The role of this part is to endure the environment conditions, at vibrations and the loads which will appear during the functionality.
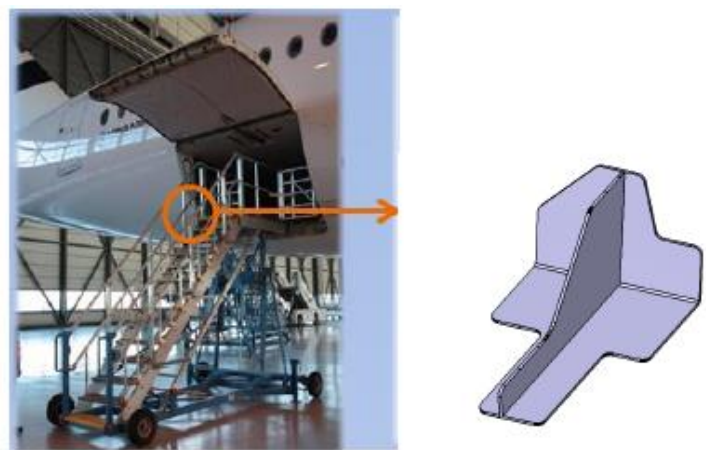

Fig. 2. Considered location of the part [10]
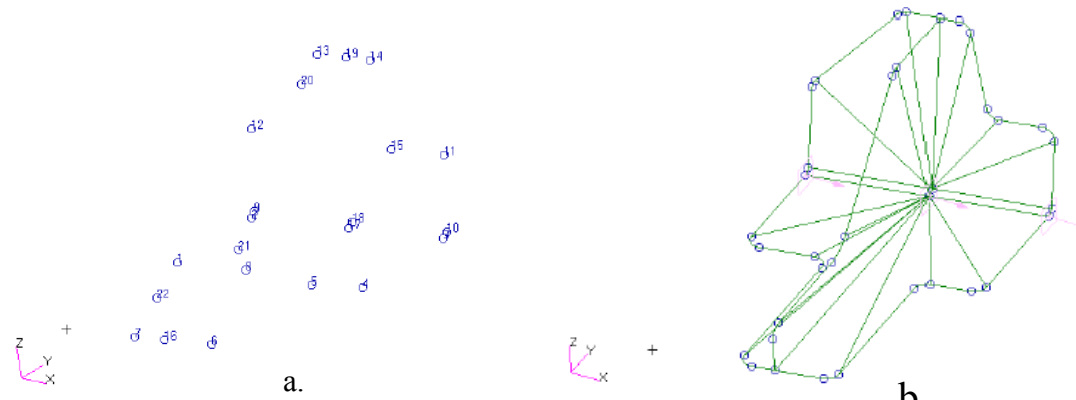

b.

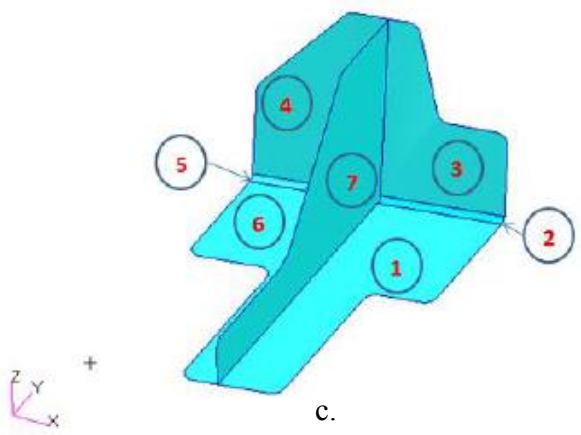

Fig. 3. Mid-Surface part creation - with nominal dimensions

This part is considered to be an element from the base project, with many loops of optimisation.

The parameterisation process leads with a CAD modelling in Catia, with the base dimensions. In this step the geometry is studied - fillets locations, angles between surfaces, 
thicknesses and also the geometric limitations for modelling. The points are extracted and imported in MSC Patran Software, creating the part with the initial proposed dimensions. The process starts with creating the points from the middle surface of the part. The location of each point is calculated considering the global coordinate system (Fig.3.a), proposing mathematical solutions for the location in space for each point; in this point the dimensional parameters are established. The final part creation will be performed by creating the established curves between the created points (Fig.3.b), surfaces between the created curves and merging the surfaces (Fig.3.c). The process is similar also for the part with thickness. The difference is that between surfaces, solids are created, which finally will comply the geometry of the part (Fig.3).

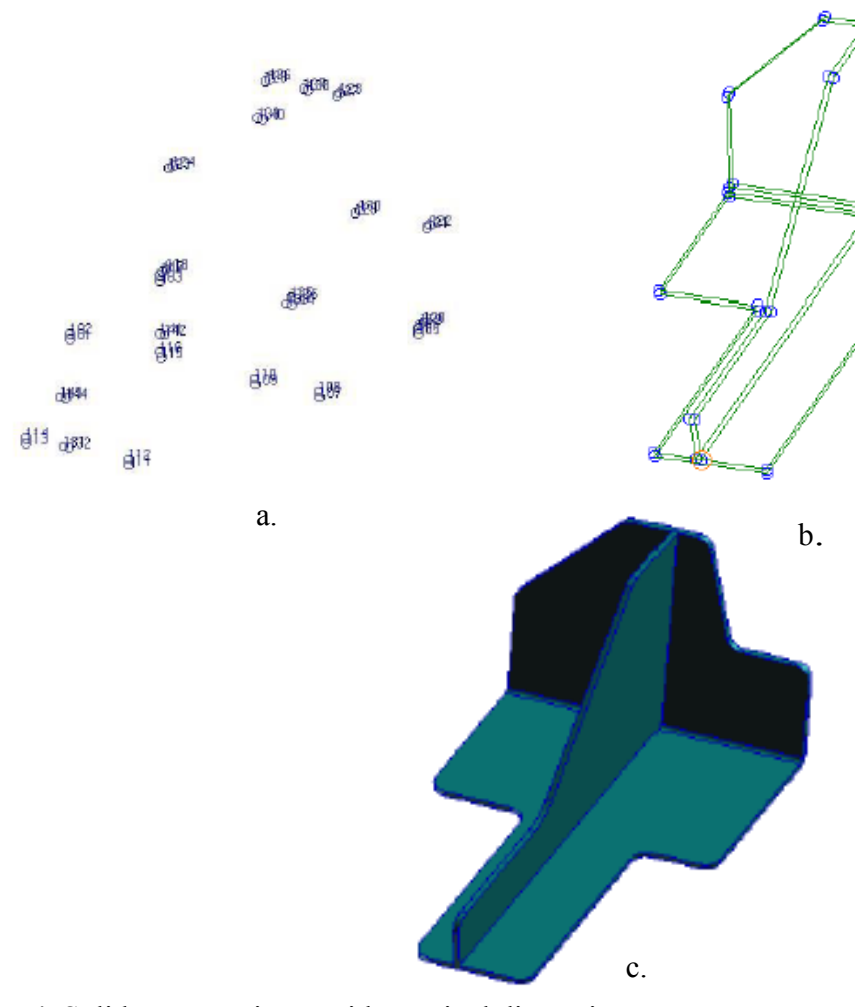

Fig. 4. Solid part creation - with nominal dimensions

After finishing the modelling in MSC Patran, the code which is registered behind the software tool is modified and adapted for Visual Basic code where the parameters are defined with mathematical relations. Also, the GUI is defined in order to establish the input values.

Using the same method is also realised the meshing. During the process of meshing for parts with initial dimensions, the errors which can appear during this phase are defined and coded for solving. For example, premeshing for a uniform element distribution is presented in Fig.5. In this case, a limitation is imposed - to use shell elements up to $4 \mathrm{~mm}$ - user requirements after tests. As parameters the user can choose the element type and its dimensions - if the values are not under the limitations, a message will be written for the user in order to choose a value from the specified range.

The adapted PCL programs into the Visual Basic language are incorporated into the software tool in order to have possibility of creating a new family of similar parts and also direct meshed parts. The software tool has also a description which helps the user to 
understand the functionality of the commands, but also it has a description of the part and the assembly in order to understand the functionality of the part and the assembly and the expectations of the project. The application output files are *.txt files which are converted in *.ses files by changing the extension and the format of the text in ASCII code. The output files can be imported in Patran and the new configuration of parts are displayed.
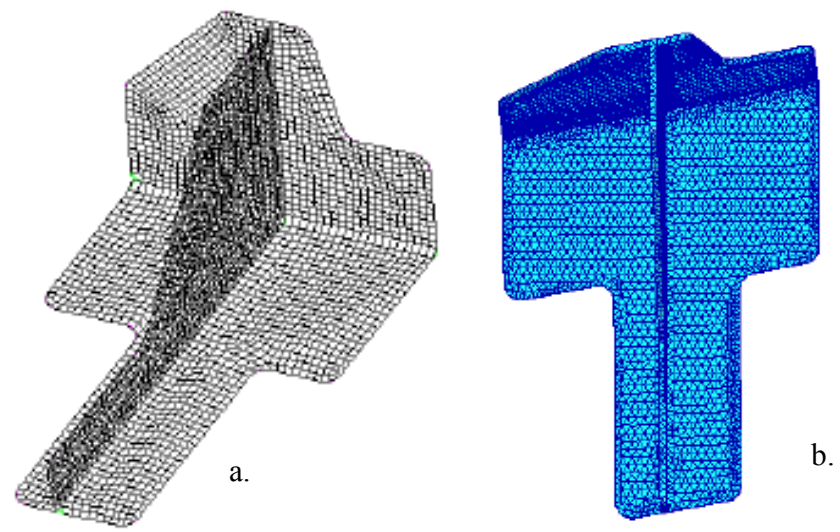

Fig. 5. 2D Shell elements meshing - elements distribution on surfaces - $2 \mathrm{~mm}$ CQUAD elements

\section{Conclusion}

The parametric design is a successful application of multi-disciplinary knowledge, being used in different industry fields. In this paper is presented a case study of a complex geometric part from the aerospace industry using PCL - Patran Command Language, which is transposed into an interface written in Visual Basic. The authors emphasized the main steps for a parametric tool development for the design and meshing phases of a part, from the part design, manufacturing and using requirements, to software limitations and to the final tool script, with a user friendly interface. As a result, this parametric design application demonstrates its suitability for mid-term and long-term projects, with a wide range of optimization processes which lead to obtain immediate qualitative results.

\section{References}

1. V. Spasojević Brkić, M. Klarin, S. Stanisavljev, A. Brkić, Z. Sajfert, South African Journal of Industrial Eng. 27, 178 (2016)

2. V. A. Deshpande, M. Prajapati, International Journal of Industrial Eng. 2, 208 (2015)

3. S. Gudnundsson, General Aviation Aircraft Design (Elsevier, 2014)

4. G.P. Monizza, D. T. Matt, C. Benedetti, Journal of Civil Engineering and Architecture 8, 1 (2014)

5. H.K. Abdullah, J.M. Kamara, American Society of Civil Engineers Proceedings (Architectural Engineering Conference 2013) 333 (2013)

6. V. Mahesh, International Conference on Advanced Materials Manufacturing, Thermal science (2013)

7. E.I. Croitoru, G. Oancea, N. Constantin, Materiale Plastice, 54, 700 (2017)

8. E.I. Croitoru, D. Magurian, G. Oancea, Transations of Famena, 40, 111, (2016)

9. A. Kosmaras, D. Tzetzis, P. Kyratsis, Academic Journal of Manufacturing Engineering, 15, 67 (2017)

10. https://www.aircraftdocking.com/products/cargo-door-stand-a350, accessed 20.07.19 